
$\$$ Research Square
Preprints are preliminary reports that have not undergone peer review.
They should not be considered conclusive, used to inform clinical practice,
or referenced by the media as validated information.

\title{
99mTc-Galacto-RGD2 Integrin av $\beta 3$ Targeted Imaging as a Surrogate for Molecular Phenotyping in Lung Cancer: Prospective Study in the Real World
}

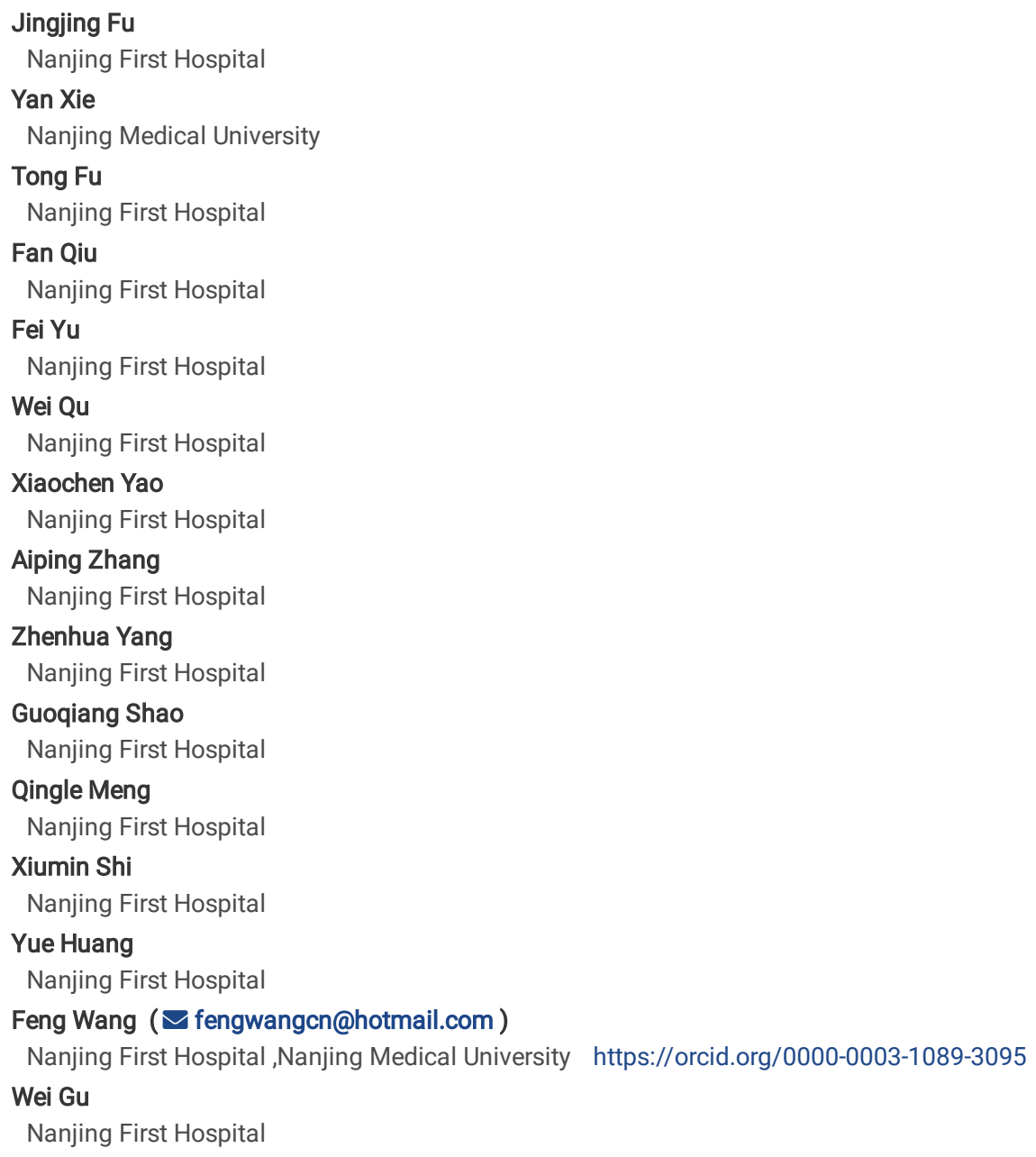




\section{Abstract}

Purpose: Epidermal growth factor receptor tyrosine kinase inhibitors (TKIs) are beneficial in patients with lung cancer. We explored the clinical value of ${ }^{99 \mathrm{~m}} \mathrm{Tc}-$ Galacto-RGD 2 single-photon emission computed tomography (SPECT/CT) in patients with lung cancer, Integrin $\mathrm{a}_{\mathrm{v}} \beta_{3}$ expression, and neovascularization in lung cancer subtypes was also addressed.

Methods: A total of 185 patients with lung cancer and 25 patients with benign lung diseases were enrolled in this prostective study from January 2013 to December 2016. All patients underwent ${ }^{99} \mathrm{~m}$ Tc-Galacto- $\mathrm{RGD}_{2}$ imaging. The region of interest was drawn around each primary lesion, and tumour uptake of

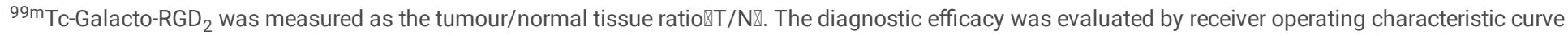
analysis. Tumour tissues were obtained from 66 patients with malignant diseases and seven with benign disease. Tumour expression levels of $a_{v} \beta_{3}$, CD31, Ki67 , and CXCR4 were analysed to determine their value for phenotyping and metastasis potential evaluation.

Results: The lung cancer patients included 22 cases of small cell lung cancer (SCLC), 48 squamous cell carcinoma (LSC), 97 adenocarcinoma (LAC), and 18 other types of lung cancer. The sensitivity, specificity, and accuracy of ${ }^{99 m}$ Tc-Galacto-RGD 2 SPECT/CT using a cut-off value of 2.5 were $91.89 \%, 48.0 \%$, and $86.67 \%$, respectively. Integrin $a_{v} \beta_{3}$ expression was higher in non-SCLC compared with SCLC, while LSC showed denser neovascularization and higher integrin

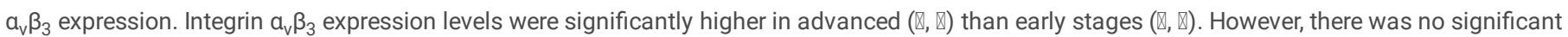
correlation between tumour uptake and $\mathrm{a}_{\mathrm{v}} \beta_{3}$ expression.

Conclusion: ${ }^{99 m}$ Tc-Galacto-RGD 2 SPECT/CT has high sensitivity but limited specificity for detecting primary lung cancer. RGD imaging may help evaluate the biological behaviour and phenotyping, and thus aid management in lung cancer.

\section{Introduction}

Lung cancer is the leading cause of cancer mortality worldwide for both sexes combined [1, 2]. The incidence and mortality of lung cancer in China have increased rapidly in the last three decades, associated with increases in air pollution and tobacco consumption [3, 4]. However, new clinical treatment strategies, such as antiangiogenic epidermal growth factor receptor-tyrosine kinase inhibitors (EGFR-TKIs) and immunotherapy, have significantly improved the outcomes of patients with lung cancer in the last decade [5]. TKIs have a cytostatic effect on tumour cells by slowing their growth and preventing the development of distant metastases [6, 7]. Multiplex genetic sequencing has been used to select appropriate TKIs, based on the recommendation of the American Society of Clinical Oncology (ASCO); however, this requires obtaining enough tumour tissue by biopsy or surgery. Unfortunately, suitable tumour specimens are unavailable for some patients due to the tumour heterogeneity or undetermined primary lesion, which limits the application of TKIs.

Nuclear medicine and molecular imaging are important for the quantitative evaluation of membrane receptors and biological tumour behaviour [8-10]. Positron emission tomography/computed tomography (PET/CT) can serve as a useful tool for identifying specific biological behaviours. ${ }^{18} \mathrm{~F}-$ fluorodeoxyglucose (FDG)-PET has also been well-validated for the diagnosis, staging, and management of malignant tumours, and can guide the selection of suitable treatment strategies $[11,12]$. However, tumour uptake of FDG is influenced by various factors, including blood glucose levels, tumour size,inflammation, and the acquisition protocol. Furthermore, FDG uptake by slow-growing and less metabolically active tumours, including highly differentiated lung cancers, was indistinguishable from that in normal tissues [13].

Angiogenesis plays important roles in tumour initiation, development, and metastasis [14]. Integrins are a diverse family of glycoproteins that form heterodimeric receptors for extracellular matrix molecules [15-17], of which integrin $a_{v} \beta_{3}$, with an exposed arginine-glycine-aspartate (RGD) tripeptide sequence, is the most-extensively studied [17]. Integrin $a_{v} \beta_{3}$ is highly expressed in the neovasculature in solid tumours, including neuroblastoma, osteosarcoma, glioblastoma, breast cancer, and prostate cancer $[10,18-25]$. The highly restricted expression of integrin $a_{v} \beta_{3}$ in normal tissues compared with its overexpression in tumour cells suggests that it may provide an interesting molecular target for the early detection of malignant tumours [18] Overexpression of integrin $a_{v} \beta_{3}$ was also correlated with tumour invasiveness in breast cancer, indicating a possible role in evaluating metastatic potential [24].

Radiolabelled RGD peptide as a target ligand for angiogenesis imaging has been well documented in preclinical and clinical studies [18, 26, 27]. In a previous multicentre study, we showed that Tc-labelled RGD dimers, such as ${ }^{99 m} \mathrm{Tc}-3 \mathrm{PRGD}$, had high sensitivity for the detection of lung cancer, including primary and metastatic tumours $[26,28,29]$. ${ }^{99 m} \mathrm{Tc}-G$ alacto-RGD 2 , with higher affinity to $\mathrm{a}_{\mathrm{v}} \beta_{3}$ and a favourable biodistribution, has been synthesized and utilized for the quantitative evaluation of $a_{v} \beta_{3}$ expression and of tumour angiogenesis, which may in turn serve as a prognostic hallmark and may aid treatment strategy selection [30].

In the clinical, multiple lymphadenopathy and remote metastasis was developed rapidly in higher aggressive lung cancer even with radical resection and comprehensive treatment, we suppose some key molecules medicate the tumour development and metastasis. Therefore, we conducted a longitudinal study to evaluate the clinical role of ${ }^{99 \mathrm{~m}} \mathrm{Tc}-\mathrm{Galacto}-\mathrm{RGD}_{2}$ SPECT/CT in a large population of patients with lung neoplasms. We also explored the expression of integrin $a_{v} \beta_{3}$ protein in tumour cells and in the neovasculature, and determined the capability of the technique to detect lymphadenopathy and bone metastasis in patients with advanced lung cancer. Herein, we investigated the value of RGD-based imaging as a surrogate for molecular phenotyping in lung cancer, and its potential use for selecting the appropriate treatment strategy, the schema of study was shown in (Fig. 1).

\section{Materials And Methods}




\section{Patients}

This prospective, single-centre study enrolled patients referred to our centre with suspected lung neoplasms from January 2013 to December $2016 .{ }^{99 m}$ TcGalacto-RGD 2 SPECT-CT was performed in all patients. Written consent was obtained from all patients, and the study was approved by the local ethics committee of Nanjing Medical University. The final diagnosis was confirmed by histopathology based on acupuncture biopsy or surgery. A total of 210 consecutive patients (147 male, 63 female; mean age $63.80 \pm 10.51$ years, range $21 \sim 85$ years) were enrolled and analysed. Of the 210 patients, 185 were confirmed with lung cancer and the other 25 patients had benign pulmonary diseases and served as the control. Patients who had undergone perioperative chemotherapy or radiotherapy were excluded from this study.

\section{Procedures}

\section{${ }^{99 m}$ Tc-Galacto-RGD 2 Radiolabelling and Quality Control}

${ }^{99 m}$ Tc-Galacto-RGD 2 labelling was carried out as described previously [30]. The Galacto-RGD 2 was friendly offered by the School of Health Sciences, Purdue University, West Lafayette, Indiana 47907, United States. Chemicals were purchased from Sigma-Aldrich (St. Louis, $\mathrm{MO}^{\mathrm{O}}$. $\mathrm{Na}^{99 \mathrm{~m}} \mathrm{TcO}_{4} \mathrm{Was}$ obtained from Dongcheng Ams Pharmaceutical (Nanjing, China). Briefly, radiolabelling with performed using a lyophilized kit formulation containing $20 \mu \mathrm{g}, 7 \mathrm{mg}$ TPPTS (trisodium triphenylphosphine-3,3',3"-trisulfonate), $6.5 \mathrm{mg}$ tricine, $40 \mathrm{mg}$ mannitol, $38.5 \mathrm{mg}$ disodium succinate hexahydrate, and $12.7 \mathrm{mg}$ succinic acid. ${ }^{99 \mathrm{~m}}$ Tclabeling was accomplished by adding $1 \sim 1.5 \mathrm{~mL}$ of $\mathrm{Na}^{99 \mathrm{~m}} \mathrm{TcO}_{4}$ solution $(1,110 \sim 1,850 \mathrm{MBq})$. The reconstituted vial was heated at $100^{\circ} \mathrm{C}$ for $30 \mathrm{~min}$ and the resulting solution was analysed by radio-high-performance liquid chromatography using a Lab Alliance system equipped with a ram IN-US detector and Zorbax C18 column ( $4.6 \mathrm{~mm} \times 250 \mathrm{~mm}, 300 \AA$ A pore size, Waters Xbridge C18, Milford, MA). The flow rate was $1 \mathrm{~mL} / \mathrm{min}$, the mobile phase was isocratic with $90 \%$ solvent $\mathrm{A}$ ( $25 \mathrm{mM} \mathrm{NH}_{4} \mathrm{OAc}$ buffer, $\mathrm{pH} 6.8$ ) and $10 \%$ solvent $\mathrm{B}$ (acetonitrile) at $0 \sim 5 \mathrm{~min}$, followed by a gradient mobile phase from $10 \% \mathrm{~B}$ at 5 min to $40 \%$ $\mathrm{B}$ at $20 \mathrm{~min}$. The radiochemical purity was $>95 \%$ for all imaging.

\section{9mTc-Galacto-RGD2 Imaging and Interpretation}

The radiochemical purity was $95.1 \% \pm 2.9 \% .{ }^{99 \mathrm{~m}} \mathrm{Tc}$-Galacto- $\mathrm{RGD}_{2}$ was administered at $555 \sim 740 \mathrm{MBq}(15 \sim 20 \mathrm{mCi})$ and whole-body images were acquired at $1 \mathrm{~h}$ post-injection. The chest image, including the upper abdomen and adrenal glands, was performed using a combined transmission and emission device with x-ray tube and detector (Symbia T6 SPECT/CT; Siemens AG, Germany). Anatomic CT images were produced for attenuation correction and tumour localization. If unexpected lesions were detected by whole-body imaging, additional abdomen or pelvis images were also acquired.

All images were interpreted independently on the computer monitor in three orthogonal planes by nuclear medicine physicians and a radiologist who were unaware of the clinical information and other imaging examinations. Significantly greater local uptake of ${ }^{99 m} \mathrm{Tc}-\mathrm{Galacto}^{-R G D} \mathrm{D}_{2}$ compared with the adjacent surrounding lung was interpreted as demonstrating a malignant lesion, and uptake less than or equal to the adjacent or surrounding lung was interpreted as a benign lesion. Focal activity in the hilum and mediastinum greater than the surrounding mediastinal activity was interpreted as lymphadenopathy. Regions of interest (ROI) were drawn around the primary lesion and contralateral lung tissue, respectively, and ${ }^{99 m} \mathrm{Tc}-\mathrm{Galacto} \mathrm{RGD}_{2}$ uptake was measured and expressed as the tumour/normal tissue ratio $(\mathrm{T} / \mathrm{N})$.

\section{Composite Reference Standard}

All available cytologic, histologic, follow-up, and imaging findings were used as a composite reference standard for the presence of tumour lesions. This is considered the optimal gold standard because cytologic or histologic verification of every lesion was not feasible or justifiable in these patients. Whenever possible, new findings on ${ }^{99 \mathrm{~m}} \mathrm{Tc}-\mathrm{Galacto}-\mathrm{RGD}_{2}$ SPECT-CT were verified by additional investigations.

\section{Immunohistochemistry (IHC) Analysis}

Tumour specimens were obtained from patients who underwent complete resection or biopsy. The sections were fixed in formalin, embedded in paraffin, deparaffinised, and stained with hematoxylin and eosin (H\&E). Integrin $a_{v} \beta_{3}, K i-67, C X C R 4$, and CD31 expression were analysed by IHC to evaluate the biological tumour behaviour. Sections were cut at 3- $\mu \mathrm{m}$, dewaxed in xylene, and rehydrated in graded ethanols. Integrin $\mathrm{a}_{\mathrm{v}} \beta_{3}$ and $\mathrm{CXCR} 4$ expression, microvascular density (CD31), and tumour cell proliferation (Ki-67) were detected by incubating the slides with monoclonal antibodies against human integrin $a_{v} \beta_{3}$ (1:200, sc-7312; Santa Cruz Biotechnology, Santa Cruz, California, US), CXCR4 (1:100, ab227767; Abcam, Massachusetts, US), Ki-67 (1:100, ab270650; Abcam), or CD31 (1:50, ab28364; Abcam), respectively, overnight, followed by horseradish peroxidase-conjugated anti-mouse IgG (1:1000, Earth Ox, Millbrae, California, US) with 3'3-diaminobenzidine as the chromogen. H\&E staining was also performed. All images were obtained at 100x magnification with the same exposure time. Brightness and contrast were adjusted similarly in all images. Integrin $\mathrm{a}_{\mathrm{v}} \beta_{3}$ and $\mathrm{CXCR} 4$ expression levels were quantified by determining the optical density (OD) after immunostaining.

\section{Statistical Analysis}

All statistical analyses were carried out using R (version 3.6.1) and graphs were constructed using GraphPad Prism software. Continuous variables with a nonnormal distribution were expressed as median (interquartile range). Differences in T/NT and protein expression levels among groups were compared using Wilcoxon's rank-sum or Kruskal-Wallis tests. The sensitivity, specificity, area under the curve (AUC), and cut-off value of T/NT were evaluated by receiver operating characteristic curve (ROC) analysis. Correlations between continuous variables with non-normal distributions were evaluated by Spearman's rank correlation analysis. Bonferroni's correction was applied for multiple comparisons. Statistical significance was established at $p<0.05$. 


\section{Results}

\section{Patient Characteristics}

The clinical characteristics of the patients are shown in Table 1. Of the 210 consecutive patients enrolled in this study, 185 ( $88.1 \%$ ) had malignant neoplasms identified by histopathology, including 22 patients with small cell lung cancer (SCLC), 97 with adenocarcinoma (LAC), 48 with squamous cell carcinoma (LSC), and 18 patients with other malignant lung tumours. Tumour tissues were obtained during thoracic surgery $(n=118)$, fine-needle aspiration $(n=35)$, or bronchoscopy $(n=32)$. Of the 25 patients with benign respiratory diseases, the benign nature of the lesion was confirmed during clinical follow-up in 12 patients, by histopathology in 7 patients, and at imaging follow-up in 6 patients. According to the Tumour, Node, and Metastasis (TNM) classification of lung cancer 8th edition published in 2015 [31], 37 patients were diagnosed with stage $\otimes(20.00 \%), 13$ with stage $\otimes(7.03 \%), 40$ with stage $\otimes(21.62 \%)$, and 95 patients with stage $\otimes(51.35 \%)$. The volume of the primary tumour (median (interquartile range): $28.01(12.30,76.33)$ was significantly higher in patients with malignant compared with benign disease $(10.89(8.66,15.77))$ (Wilcoxon's rank-sum test, $\left.p=1.69 \times 10^{-4}\right)$.

Table 1

Clinical characteristics of 210 subjects

\begin{tabular}{|c|c|c|c|}
\hline Variants & Lung cancer & Benign disease & $p$ \\
\hline \multicolumn{4}{|l|}{ General } \\
\hline Age & $64.17 \pm 10.15$ & $61.04 \pm 12.77$ & 0.25 \\
\hline \multicolumn{4}{|l|}{ Sex } \\
\hline Male & $133(56.00 \%)$ & 14(71.89\%) & \\
\hline Female & $52(44.00 \%)$ & $11(28.11 \%)$ & 0.16 \\
\hline \multicolumn{4}{|c|}{ Cancer Type } \\
\hline LAC & $97(52.43 \%)$ & / & \\
\hline LSC & $48(25.95 \%)$ & I & \\
\hline SCLC & $22(11.89 \%)$ & / & \\
\hline Other & $18(9.73 \%)$ & / & \\
\hline \multicolumn{4}{|l|}{ Stage } \\
\hline ૫ & $37(20.00 \%)$ & / & \\
\hline ૫ & $13(7.03 \%)$ & / & \\
\hline प & $40(21.62 \%)$ & / & \\
\hline ( & $95(51.35 \%)$ & / & \\
\hline
\end{tabular}

\section{9m Tc-Galacto-RGD2 Imaging and Interpretation}

High-contrast images acquired $1 \mathrm{~h}$ after injection of ${ }^{99 \mathrm{~m}} \mathrm{Tc}-$ Galacto- $\mathrm{RGD}_{2}$ showed higher focal uptake in malignant primary tumours and metastatic lymph nodes (Fig. 2), compared with significantly lower uptake in benign lesions (T/NT in malignant diseases: 6.84 (4.62, 9.86); benign diseases: 2.53 (1.24, 3.91); $p$

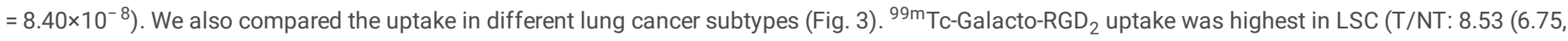
10.99)), followed by LAC (T/NT: $6.84(4.64,9.07))$ and SCLC (T/NT: $4.73(2.47,5.85))$. Other types of lung cancer (T/NT: 5.23 (3.32, 11.50)) showed moderate radioactivity in the primary tumour, with no significant difference between other types and LSC, LAC, and SCLC. We also compared uptake by the primary

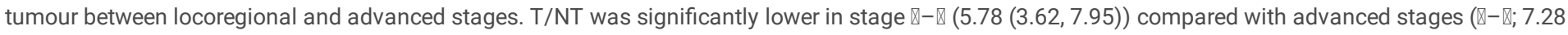
$(5.43,10.34))\left(p=1.56 \times 10^{-3}\right)$. However, there was overlap with inflammatory pseudotumours, tuberculosis. RGD avidity was found in rare case with pulmonary sequestration and thymoma, respectively, due to higher density of micro-vessels (Figs. 4, 5). ROC analysis indicated that the sensitivity, specificity, and accuracy of ${ }^{99 \mathrm{~m}}$ Tc-Galacto-RGD 2 were $91.89 \%, 48.0 \%$, and $86.67 \%$, respectively, using a cut-off value of 2.5 . With a T/NT cut-off value of 3.94 , the AUC was 0.83 and the sensitivity and the specificity were $82.7 \%$ and $76.0 \%$, respectively.

\section{Histopathology and IHC}

Of the 210 patients with suspected lung cancer, immunochemistry was performed in 66 patients with lung cancer and seven patients with benign diseases. Expression levels of integrin $a_{v} \beta_{3}$ were significantly higher in tissues from patients with lung cancer (OD: 15,020.5 (4482.6, 44,455.2)) compared with benign diseases (OD: 1797.8 (794.0, 2943.6); $p=1.08 \times 10^{-3}$ ) (Table 2). CD31 levels were also elevated in lung cancer (OD: 21.9 (13.75, 34.35) vs 9.00 (8.90, 11.50); $\left.p=5.56 \times 10^{-3}\right)$. Higher levels of integrin $\mathrm{a}_{\mathrm{v}} \beta_{3}$ were expressed in advanced tumours (OD: 19,729.00 $\left.(6445.40,45,288.30)\right)$ compared with locoregional tumours $(5914.40(1461.60,17,658.20)), p=3.10 \times 10^{-2}$. Integrin $a_{v} \beta_{3}$ was also highly expressed not only in endothelial cells in the neovasculature, reflected by CD31 expression, but also in tumour cells (Fig. 5), with a higher density of neovasculature and integrin $\mathrm{a}_{\mathrm{v}} \beta_{3}$ expression in the primary tumour. Integrin $\mathrm{a}_{\mathrm{v}} \beta_{3}$ was also significantly correlated with CD31 expression in lung cancer $(r=0.30, p=0.016)$. However, there was no correlation between tumour uptake of $99 \mathrm{~m}$ Tc- 
Galacto-RGD 2 and integrin $a_{v} \beta_{3}$ expression in the primary tumour in this study (Fig. supplemewnt-1). Squamous lung cancer usually showed higher level of $a_{v} \beta_{3}$ in the tumor cell and the higher density of microvessel, which was consistent with RGD imaging as shown in the (Fig. Supplement-2). Aggressive LAC tends to higher express integrin $a_{v} \beta_{3}$ in the tumour cell and has more dense microvessel, which showed focal uptake in the RGD image, as shown in the (Fig. Supplement-3). Neo-vascularization varied in benign respiratory diseases, associated with higher integrin $a_{v} \beta_{3}$ expression. In the current study, integrin $a_{v} \beta_{3}$ correlated with CD31 expression in the neo-vessel, indicating that integrin $\mathrm{a}_{\mathrm{v}} \beta_{3}$ mediated angiogenesis, leading to tumour development and metastasis. We also examined CXCR4 expression. CXCR4 was highly expressed in lung cancer, as demonstrated by IHC. Furthermore, expression levels of CXCR4 tended to be positively correlated with integrin $\mathrm{a}_{\mathrm{v}} \beta_{3}$ levels in lung cancer specimens $(r=0.22, p=0.08)$. In addition, the proliferation index (Ki-67) in LSC and SCLC (27.45 $(11.88,42.00)$ and $70.00(55.13,73.48)$, respectively) were both significantly higher than in LAC $(10.15(2.98,27.89))($ Table 3$)$.

Table 2

Immunohistochemical results of lung cancer and benign disease[

\begin{tabular}{|llll|}
\hline Variants & Lung cancer $(\boldsymbol{n}=\mathbf{6 6})$ & Benign disease $(\boldsymbol{n}=\mathbf{7})$ & $\boldsymbol{p}$ \\
\hline Integrin avß3 & $15020.5(4482.6,44455.2)$ & $1797.8(794.0,2943.6)$ & $\mathbf{1 . 0 8 E - 0 3}$ \\
\hline CXCR4 & $5120.0(1978.0,18460.0)$ & $538.6(300.0,7101.7)$ & 0.08 \\
CD31 & $21.9(13.75,34.35)$ & $9.00(8.90,11.50)$ & $\mathbf{5 . 5 6 E - 0 3}$ \\
\hline Ki-67 & $20.00(7.46,40.00)$ & $/$ & \\
\hline
\end{tabular}

Table 3

Immunohistochemical results among different groups.

\begin{tabular}{|c|c|c|c|c|c|c|c|}
\hline & \multicolumn{3}{|l|}{ Stage } & \multicolumn{4}{|l|}{ Class } \\
\hline & 囚 】 & 凶 \ & $P$ & LAC & LSC & SCLC & $P$ \\
\hline & $(n=21)$ & $(n=45)$ & & $(n=34)$ & $(n=26)$ & $(n=6)$ & \\
\hline $\begin{array}{l}\text { Integrin } \\
\text { av } \beta 3\end{array}$ & $\begin{array}{l}\text { 5914.4(1461.6, } \\
17658.2)\end{array}$ & $\begin{array}{l}\text { 19729.0(6445.4, } \\
45288.3)\end{array}$ & $\begin{array}{l}3.10 \mathrm{E}- \\
02\end{array}$ & 17308.1(7973.6,47755.9) & $9721.6(3186.5,23010.7)$ & $6485.0(3083.0,28119.0)$ & 0. \\
\hline CXCR4 & $8438.0(2582.5,22821.8)$ & $4002(1863,11541)$ & 0.18 & $4536(1348,10501)$ & $9052.0(3688.9,33442.9)$ & 2324(1980,4395) & 0. \\
\hline CD31 & $28.20(14.25,34.40)$ & $21.33(13.75,32.00)$ & 0.54 & $27.80(14.44,34.35)$ & 19.72(15.06,32.11) & $13.32(11.75,20.04)$ & 0. \\
\hline Ki67 & $11.00(5.00,40.00)$ & $22.82(8.63,43.95)$ & 0.47 & $10.15(2.98,27.89)$ & $27.45(11.88,42.00)$ & $70.00(55.13,73.48)$ & 5. \\
\hline
\end{tabular}

Abbreviation: LAC adenocarcinoma, LSC squamous cell carcinoma, SCLC small cell lung cancer

\section{Lymphadenopathy and Distant Metastasis}

Of the 185 patients with lung cancer, 116 patients had lymphadenopathy, 87 had remote metastasis, 17 had multiple lung tumours including pleural invasion, and 70 patients had bone metastasis. The metastatic lymph nodes and remote metastases showed high focal uptake of ${ }^{99 m} \mathrm{Tc}-\mathrm{Galacto}_{\mathrm{RGD}}$. However, although lymphadenopathy was evaluated by imaging follow-up, the final diagnosis was not confirmed, and we were therefore unable to evaluate the diagnostic value of ${ }^{99 \mathrm{~m}} \mathrm{Tc}-\mathrm{G}$ alacto- $\mathrm{RGD}_{2}$ imaging for lymphadenopathy and remote metastasis in this study.

\section{Discussion}

Targeted therapy has significantly improved the outcome for patients with lung cancer in the last decade [32]. For example, EGFR is a major driver of NSCLC tumorigenesis [33], and tumour growth can be inhibited by treating lung tumours expressing somatic mutations of the EGFR gene with TKIs. This strategy revealed the potential for precise biomarker-directed and personalized treatments for lung cancer [34, 35]. However, EGFR status is determined by histological tumour biopsy, and it is not always possible to obtain a representative biopsy suitable for precise histopathology because of tumour localization and heterogeneity, and small tumour specimens. Selecting TKI-sensitive patients thus remains a challenge, highlighting the need for alternative (preferably noninvasive) means of patient selection. We previously validated the ability of ${ }^{99 m} \mathrm{Tc}-$ Galacto-RGD 2 to identify iodine-refractory status in patients with thyroid cancer [36]. In a rare case with a solitary fibrous tumour located in the main pulmonary artery, ${ }^{99 \mathrm{~m}} \mathrm{Tc}-\mathrm{Galacto}^{-\mathrm{RGD}_{2}}$ imaging played an important role in detecting the primary tumour and predicting the metastatic potential [27]. In the current study, we evaluated the use of ${ }^{99 m}$ Tc-Galacto-RGD 2 SPECT/CT for the detection of lung cancer. We also explored the expression of integrin $a_{v} \beta_{3}$ and CXCR4 in different lung cancer subtypes, and compared the neovasculature among these subtypes. We also examined the correlations between tumour uptake of ${ }^{99 m} \mathrm{Tc}-\mathrm{Galacto} \mathrm{RGD}_{2}$ and integrin $\mathrm{a}_{\mathrm{v}} \beta_{3}$ expression and neovascularization. Finally, we validated the use of integrin molecular imaging as a surrogate for phenotyping.

High-contrast images acquired $1 \mathrm{~h}$ after injection of ${ }^{99 \mathrm{~m}} \mathrm{Tc}$-Galacto-RGD2 showed a significantly higher T/NT ratio in malignant compared with benign lung lesions. Malignant primary tumours and metastatic lymph nodes showed higher focal uptake, while benign lesion showed significantly lower uptake. ${ }^{99 m} \mathrm{Tc}$ Galacto-RGD 2 SPECT/CT showed high sensitivity for detecting primary tumours and remote metastases. ROC analysis showed a sensitivity and accuracy of $91.89 \%$ and $86.67 \%$, respectively, for ${ }^{99 m}$ Tc-Galacto-RGD 2 SPECT/CT, using a cut-off value of 2.5 . However, the specificity for differentiating between malignant and benign disease was limited, possibly because of the involvement of integrin $\mathrm{a}_{\mathrm{v}} \beta_{3}$ in various benign diseases. Overlap usually occurs between 
tuberculosis and inflammatory pseudo-tumours, which usually show higher uptake of ${ }^{99 \mathrm{~m}} \mathrm{Tc}-\mathrm{Galacto}^{-R G D_{2}}$ than other types of benign diseases, such as pneumonia [18].

In the current study, IHC showed that $\mathrm{a}_{\mathrm{v}} \beta_{3}$ levels were higher in advanced lung cancer, and proliferation index, represented by Ki-67, was significantly increased in advanced stages of SCLC, associated with metastatic potential $[18,24,37]$. Patients with lung cancer, even in the early stages, may develop multiple metastases several months after thorough tumour resection, possibly related to specific tumour types with higher metastatic potential. In the current study, CXCR4 expression levels were higher in lung cancer compared with benign disease, though the differences were not significant. Its expression was correlated with both integrin $a_{v} \beta_{3}$ and CD31 expression in primary lung tumours, while integrin $a_{v} \beta_{3}$ was also correlated with CD31. These findings validate our hypothesis that lymphadenopathy and remote metastasis are mediated by specific biological molecules. Integrin $\mathrm{a}_{\mathrm{v}} \beta_{3}$ and CXCR4 may mediate angiogenesis, which may further promote lymph node and remote metastases. Imaging targeting integrin $a_{v} \beta_{3}$ may thus improve our understanding of the interactions between cancer cells and their microenvironment, which is a necessary prerequisite for the development of treatment strategies specifically targeting cancerinduced invasion and metastases. This information is significant in light of the correlations of integrin $a_{v} \beta_{3}$ overexpression with recurrence and poor prognosis, and in relation to early diagnosis and treatment-response monitoring. These findings demonstrated that expression levels of integrin $a_{v} \beta_{3}$ were strongly correlated with tumorigenic and aggressive behaviours in lung cancer cells. CXCR4 has been implicated in the chemotactic migration of cancer cells [16]. CXCR4 and integrin might synergistically promote lymphatic metastasis in lung cancer, and might act as clinical predictors of lymph node metastasis in NSCLC [38-40]. High expression levels of chemokines are related to a poor prognosis and chemotherapy tolerance in cancer patients [41-44]. CXCR4 is a chemokine receptor that plays a critical role in the process of lymphocyte homing to lymphatic vessels and secondary lymphoid organs, including the lymph nodes [45].

Integrin $\mathrm{a}_{\mathrm{v}} \beta_{3}$ was expressed not only in the tumour cells, but also in the endothelium, though there was a lack of a correlation between tumour uptake of

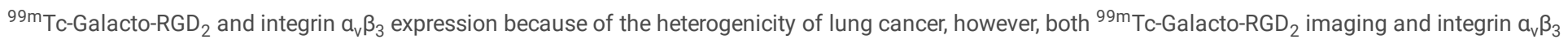
expression behaved well in distinguishing lung cancer and benign lung disease. We supposed that tumour uptake of ${ }^{99 \mathrm{~m}} \mathrm{Tc}_{\mathrm{C}}-\mathrm{Galacto}^{-R G D_{2}}$ was related to integrin $a_{v} \beta_{3}$ expression, neovascularization, and tumour stage. Integrin $a_{v} \beta_{3}$ expression in tumour cells promoted lymphatic and distant metastases, as observed (Fig. 1). However, benign diseases showed variable degrees of angiogenesis, also associated with higher expression of integrin $a_{v} \beta_{3}$, as shown in one patient with thymus adenoma and in another with pulmonary sequestration (Figs. 3, 4). We hypothesized that tumour uptake of ${ }^{99 m} \mathrm{Tc}_{\mathrm{C}} \mathrm{Galacto}_{\mathrm{B}} \mathrm{RGD}_{2}$ depended on the neovasculature and integrin $\mathrm{a}_{\mathrm{v}} \beta_{3}$ expression in the tumour cell, and focal uptake in RGD-targeted imaging would thus be higher in primary tumours with more neovasculature and higher integrin $\mathrm{a}_{\mathrm{v}} \beta_{3}$ expression. Regarding the different subtypes of lung cancer, LSC usually had more neovascularization and higher integrin $a_{v} \beta_{3}$ expression, followed by LAC, while SCLC usually showed less neovascularization and a higher proliferation index. The highest T/NT ratio was therefore found in LSC (8.53), and was significantly higher than that in LAC and SCLCs (6.84 and 4.73, respectively) (Fig. 2). RGDtargeted imaging may thus serve as a useful tool for the phenotyping of lung cancer, which will in turn be important for helping to select suitable treatment strategies.

In conclusion, this was the first extensive longitudinal study to investigate the expression of integrin $\mathrm{a}_{\mathrm{v}} \beta_{3}$ in lung cancer. ${ }^{99 \mathrm{~m}} \mathrm{Tc}-\mathrm{Galacto} \mathrm{RGD} \mathrm{D}_{2}$ imaging showed high sensitivity for the detection of primary lung cancer, but limited specificity. ${ }^{99 \mathrm{~m}} \mathrm{Tc}$-Galacto-RGD 2 uptake in the primary tumour was attributed to integrin $a_{v} \beta_{3}$ expression in the endothelial cells and tumour cells, and greater focal uptake occurred in primary lung cancers with more neovascularization and high levels of integrin $\alpha_{v} \beta_{3}$ in the tumour cells. LSC had a higher density of neo-vessels and higher integrin $a_{v} \beta_{3}$ expression, followed by LAC and then SCLC. Furthermore, advanced lung cancer showed higher expression levels of integrin $a_{v} \beta_{3}$ compared with early stages, and higher integrin $a_{v} \beta_{3}$ and $C X C R 4$ expression in the tumour cells may mediate lymphatic and distant metastases. These two molecules might thus serve as independent predictors of patient prognosis. These findings suggest that RGD based imaging might be a useful tool for lung cancer phenotyping and for evaluating tumour biological behaviours, such as aggressiveness. integrin $\mathrm{a}_{\mathrm{v}} \beta_{3}$ targeted imaging might thus be a valuable tool to aid the selection of molecular targeted treatment strategies. However, further studies are needed to validate the current findings and to address the issue that tumour specimens suitable for IHC are not obtained from all patients.

\section{Declarations}

All procedures performed in this study involving human participants were carried out in accordance with the ethical standards of the Nanjing Medical University and/or national research committee and with the 1964 Helsinki Declaration and its later amendments or comparable ethical standards. Informed consent was obtained from all patients. This research was supported by grants from the National Natural Science Foundation of China (11805104, 82003532), Jiangsu Provincial Key Research and Development Special Fund (BE2017612), Nanjing Medical Foundation (ZKX17027), Health Commission of Jiangsu Province (H2019091), Nanjing Medical and Health International Joint Research and Development Project (201911042), The Second Round Fund of Nanjing Clinical Medical Center "Nanjing Nuclear Medicine Centre". There is no conflicts of interest.

\section{Acknowledgments}

We are grateful to our colleagues for clinical data collection and analysis. We'd like to thank Prof. Shuang Liu From Health College of Purdue University for radiotracer synthesis and study design, also thank Susan Furness, PhD, from Liwen Bianji, Edanz Editing China (www.liwenbianji.cn/ac), for editing the English text of a draft of this manuscript.

\section{References}


1. Bray F, Ferlay J, Soerjomataram I, Siegel RL, Torre LA, Jemal A. Global cancer statistics 2018: GLOBOCAN estimates of incidence and mortality worldwide for 36 cancers in 185 countries. CA Cancer J Clin. 2018;68:394-424. doi:10.3322/caac.21492.

2. Chen W, Zheng R, Baade PD, Zhang S, Zeng H, Bray F, et al. Cancer statistics in China, 2015. CA Cancer J Clin. 2016;66:115-32. doi:10.3322/caac.21338.

3. Yang D, Liu Y, Bai C, Wang X, Powell CA. Epidemiology of lung cancer and lung cancer screening programs in China and the United States. Cancer Lett. 2020;468:82-7. doi:10.1016/j.canlet.2019.10.009.

4. Zhang H, Cai B. The impact of tobacco on lung health in China. Respirology. 2003;8:17-21. doi:10.1046/j.1440-1843.2003.00433.x.

5. Arbour KC, Riely GJ. Systemic Therapy for Locally Advanced and Metastatic Non-Small Cell Lung Cancer: A Review. JAMA. 2019;322:764-74. doi:10.1001/jama.2019.11058.

6. Travis WD, Brambilla E, Nicholson AG, Yatabe Y, Austin JHM, Beasley MB, et al. The 2015 World Health Organization Classification of Lung Tumors: Impact of Genetic, Clinical and Radiologic Advances Since the 2004 Classification. J Thorac Oncol. 2015;10:1243-60. doi:10.1097/JTO.0000000000000630.

7. Ferguson FM, Gray NS. Kinase inhibitors: the road ahead. Nat Rev Drug Discov. 2018;17:353-77. doi:10.1038/nrd.2018.21.

8. Mishani E, Abourbeh G. Cancer molecular imaging: radionuclide-based biomarkers of the epidermal growth factor receptor (EGFR). Curr Top Med Chem. 2007;7:1755-72. doi:10.2174/156802607782507457.

9. Sanders JC, Kuwert T, Hornegger J, Ritt P. Quantitative SPECT/CT Imaging of (177)Lu with In Vivo Validation in Patients Undergoing Peptide Receptor Radionuclide Therapy. Mol Imaging Biol. 2015;17:585-93. doi:10.1007/s11307-014-0806-4.

10. Wang T, Li G, Wang D, Li F, Men D, Hu T, et al. Quantitative profiling of integrin alphavbeta3 on single cells with quantum dot labeling to reveal the phenotypic heterogeneity of glioblastoma. Nanoscale. 2019;11:18224-31. doi:10.1039/c9nr01105f.

11. Cremonesi M, Gilardi L, Ferrari ME, Piperno G, Travaini LL, Timmerman R, et al. Role of interim (18)F-FDG-PET/CT for the early prediction of clinical outcomes of Non-Small Cell Lung Cancer (NSCLC) during radiotherapy or chemo-radiotherapy. A systematic review. Eur J Nucl Med Mol Imaging. 2017;44:1915-27. doi:10.1007/s00259-017-3762-9.

12. Iravani A, Hicks RJ. Imaging the Cancer Immune Environment and Its Response to Pharmacologic Intervention, Part 1: The Role of (18)F-FDG PET/CT. J Nucl Med. 2020;61:943-50. doi:10.2967/jnumed.119.234278.

13. Kang F, Mu W, Gong J, Wang S, Li G, Li G, et al. Integrating manual diagnosis into radiomics for reducing the false positive rate of (18)F-FDG PET/CT diagnosis in patients with suspected lung cancer. Eur J Nucl Med Mol Imaging. 2019;46:2770-9. doi:10.1007/s00259-019-04418-0.

14. Baeriswyl V, Christofori G. The angiogenic switch in carcinogenesis. Semin Cancer Biol. 2009;19:329-37. doi:10.1016/j.semcancer.2009.05.003.

15. Zaidel-Bar R. Job-splitting among integrins. Nat Cell Biol. 2013;15:575-7. doi:10.1038/ncb2770.

16. Wu D, Xu Y, Ding T, Zu Y, Yang C, Yu L. Pairing of integrins with ECM proteins determines migrasome formation. Cell Res. 2017;27:1397-400. doi:10.1038/cr.2017.108.

17. Ruoslahti E, Pierschbacher MD. New perspectives in cell adhesion: RGD and integrins. Science. 1987;238:491-7. doi:10.1126/science.2821619.

18. Niu G, Chen X. Why integrin as a primary target for imaging and therapy. Theranostics. 2011;1:30-47. doi:10.7150/thno/v01p0030.

19. Demircioglu F, Hodivala-Dilke K. alphavbeta3 Integrin and tumour blood vessels-learning from the past to shape the future. Curr Opin Cell Biol. 2016;42:121-7. doi:10.1016/j.ceb.2016.07.008.

20. Sun X, Ma T, Liu H, Yu X, Wu Y, Shi J, et al. Longitudinal monitoring of tumor antiangiogenic therapy with near-infrared fluorophore-labeled agents targeted to integrin alphavbeta3 and vascular endothelial growth factor. Eur J Nucl Med Mol Imaging. 2014;41:1428-39. doi:10.1007/s00259-014-2702-1.

21. Joseph JM, Gross N, Lassau N, Rouffiac V, Opolon P, Laudani L, et al. In vivo echographic evidence of tumoral vascularization and microenvironment interactions in metastatic orthotopic human neuroblastoma xenografts. Int J Cancer. 2005;113:881-90. doi:10.1002/ijc.20681.

22. Zou W, Teitelbaum SL. Absence of Dap12 and the alphavbeta3 integrin causes severe osteopetrosis. J Cell Biol. 2015;208:125-36. doi:10.1083/jcb.201410123.

23. Zhang L, Meng X, Shan X, Gu T, Zhang J, Feng S, et al. Integrin alphavbeta3-Specific Hydrocyanine for Cooperative Targeting of Glioblastoma with High Sensitivity and Specificity. Anal Chem. 2019;91:12587-95. doi:10.1021/acs.analchem.9b03725.

24. Wu FH, Luo LQ, Liu Y, Zhan QX, Luo C, Luo J, et al. Cyclin D1b splice variant promotes alphavbeta3-mediated adhesion and invasive migration of breast cancer cells. Cancer Lett. 2014;355:159-67. doi:10.1016/j.canlet.2014.08.044.

25. Krishn SR, Singh A, Bowler N, Duffy AN, Friedman A, Fedele C, et al. Prostate cancer sheds the alphavbeta3 integrin in vivo through exosomes. Matrix Biol. 2019;77:41-57. doi:10.1016/j.matbio.2018.08.004.

26. Yan B, Qiu F, Ren L, Dai H, Fang W, Zhu H, et al. (99m)Tc-3P-RGD2 molecular imaging targeting integrin alphavbeta3 in head and neck squamous cancer xenograft. J Radioanal Nucl Chem. 2015;304:1171-7. doi:10.1007/s10967-015-3928-5.

27. Luo R, Jiang Y, Huang Y, Chen X, Wang F. Longitudinal observation of solitary fibrous tumor translation into malignant pulmonary artery intimal sarcoma. J Cardiothorac Surg. 2020;15:233. doi:10.1186/s13019-020-01271-3.

28. Yan B, Fu T, Liu Y, Wei W, Dai H, Fang W, et al. 99mTc-3PRGD2 single-photon emission computed tomography/computed tomography for the diagnosis of choroidal melanoma: A preliminary STROBE-compliant observational study. Medicine (Baltimore). 2018;97:e12441. doi:10.1097/MD.0000000000012441.

29. Fu T, Qu W, Qiu F, Li Y, Shao G, Tian W, et al. (99m)Tc-3P-RGD2 micro-single-photon emission computed tomography/computed tomography provides a rational basis for integrin alphavbeta3-targeted therapy. Cancer Biother Radiopharm. 2014;29:351-8. doi:10.1089/cbr.2014.1622.

30. Ji S, Czerwinski A, Zhou Y, Shao G, Valenzuela F, Sowinski P, et al. (99m)Tc-Galacto-RGD2: a novel 99mTc-labeled cyclic RGD peptide dimer useful for tumor imaging. Mol Pharm. 2013;10:3304-14. doi:10.1021/mp400085d. 
31. Kay FU, Kandathil A, Batra K, Saboo SS, Abbara S, Rajiah P. Revisions to the Tumor, Node, Metastasis staging of lung cancer (8(th) edition): Rationale, radiologic findings and clinical implications. World J Radiol. 2017;9:269-79. doi:10.4329/wjr.v9.i6.269.

32. Redman MW, Papadimitrakopoulou VA, Minichiello K, Hirsch FR, Mack PC, Schwartz LH, et al. Biomarker-driven therapies for previously treated squamous non-small-cell lung cancer (Lung-MAP SWOG S1400): a biomarker-driven master protocol. Lancet Oncol. 2020;21:1589-601. doi:10.1016/S14702045(20)30475-7.

33. Tan DS, LeighI NB, Riely GJ, Yang JC, Sequist LV, Wolf J, et al. Safety and efficacy of nazartinib (EGF816) in adults with EGFR-mutant non-small-cell lung carcinoma: a multicentre, open-label, phase 1 study. Lancet Respir Med. 2020;8:561-72. doi:10.1016/S2213-2600(19)30267-X.

34. Oshima Y, Tanimoto T, Yuji K, Tojo A. EGFR-TKI-Associated Interstitial Pneumonitis in Nivolumab-Treated Patients With Non-Small Cell Lung Cancer. JAMA Oncol. 2018;4:1112-5. doi:10.1001/jamaoncol.2017.4526.

35. Cho JH, Lim SH, An HJ, Kim KH, Park KU, Kang EJ, et al. Osimertinib for Patients With Non-Small-Cell Lung Cancer Harboring Uncommon EGFR Mutations: A Multicenter, Open-Label, Phase II Trial (KCSG-LU15-09). J Clin Oncol. 2020;38:488-95. doi:10.1200/JC0.19.00931.

36. Xu Q, Liu R, Wang J, Huang Y, Li S, Zhang L, et al. Role of [(99m)Tc]Tc-Galacto-RGD2 SPECT/CT in identifying metastatic differentiated thyroid carcinoma after thyroidectomy and radioactive iodine therapy. Nucl Med Biol. 2020;88-89:34-43. doi:10.1016/j.nucmedbio.2020.06.006.

37. Hoster E, Rosenwald A, Berger F, Bernd HW, Hartmann S, Loddenkemper C, et al. Prognostic Value of Ki-67 Index, Cytology, and Growth Pattern in MantleCell Lymphoma: Results From Randomized Trials of the European Mantle Cell Lymphoma Network. J Clin Oncol. 2016;34:1386-94. doi:10.1200/JCO.2015.63.8387.

38. Chen FH, Fu SY, Yang YC, Wang CC, Chiang CS, Hong JH. Combination of vessel-targeting agents and fractionated radiation therapy: the role of the SDF1/CXCR4 pathway. Int J Radiat Oncol Biol Phys. 2013;86:777-84. doi:10.1016/j.jijrobp.2013.02.036.

39. Iwakiri S, Mino N, Takahashi T, Sonobe M, Nagai S, Okubo K, et al. Higher expression of chemokine receptor CXCR7 is linked to early and metastatic recurrence in pathological stage I nonsmall cell lung cancer. Cancer. 2009;115:2580-93. doi:10.1002/cncr.24281.

40. Katende JM, Goddeeris BM, Morzaria SP, Nkonge CG, Musoke AJ. Identification of a Theileria mutans-specific antigen for use in an antibody and antigen detection ELISA. Parasite Immunol. 1990;12:419-33. doi:10.1111/j.1365-3024.1990.tb00978.x.

41. Demir IE, Mota Reyes C. Chemokines: the (un)usual suspects in pancreatic cancer neural invasion. Nat Rev Gastroenterol Hepatol. 2020. doi:10.1038/s41575-020-0329-1.

42. Peng D, Kryczek I, Nagarsheth N, Zhao L, Wei S, Wang W, et al. Epigenetic silencing of TH1-type chemokines shapes tumour immunity and immunotherapy. Nature. 2015;527:249-53. doi:10.1038/nature15520.

43. Lau S, Feitzinger A, Venkiteswaran G, Wang J, Lewellis SW, Koplinski CA, et al. A negative-feedback loop maintains optimal chemokine concentrations for directional cell migration. Nat Cell Biol. 2020;22:266-73. doi:10.1038/s41556-020-0465-4.

44. Kwon D, Lozada J, Zhang Z, Zeisler J, Poon R, Zhang C, et al. High-Contrast CXCR4-Targeted (18)F-PET Imaging Using a Potent and Selective Antagonist. Mol Pharm. 2021;18:187-97. doi:10.1021/acs.molpharmaceut.0c00785.

45. Cabioglu N, Yazici MS, Arun B, Broglio KR, Hortobagyi GN, Price JE, et al. CCR7 and CXCR4 as novel biomarkers predicting axillary lymph node metastasis in T1 breast cancer. Clin Cancer Res. 2005;11:5686-93. doi:10.1158/1078-0432.CCR-05-0014.

\section{Figures}




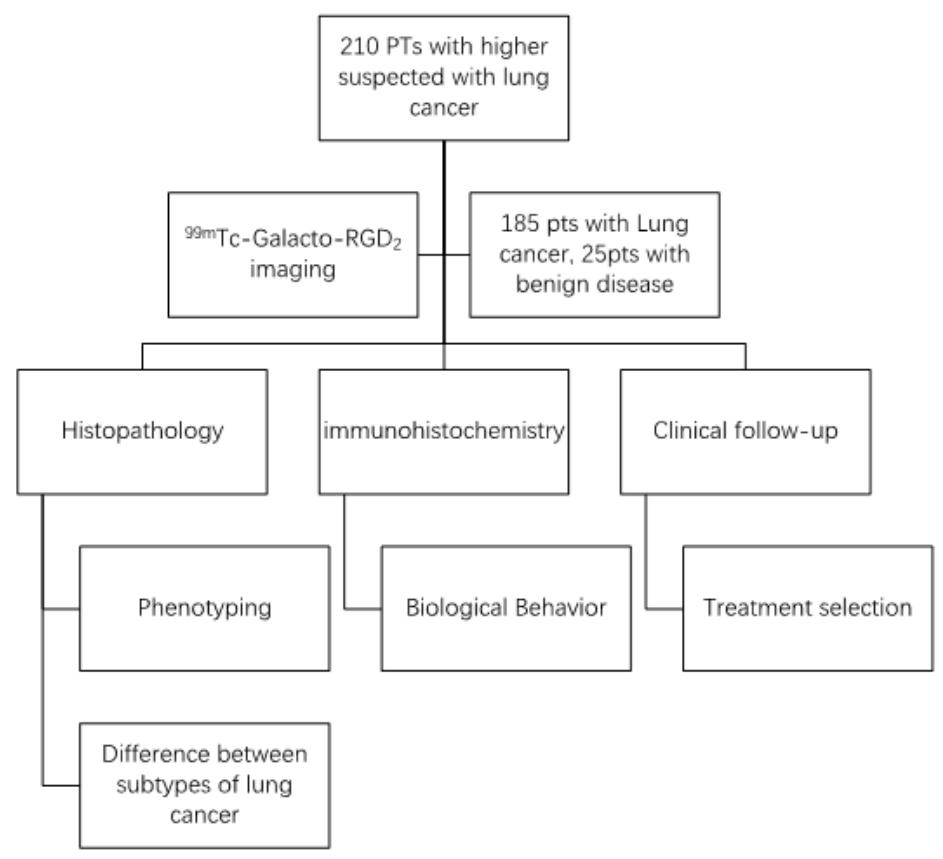

Figure 1

The study flow for the 99mTc-Galacto-RGD2 imaging in the diagnosis of lung cancer.
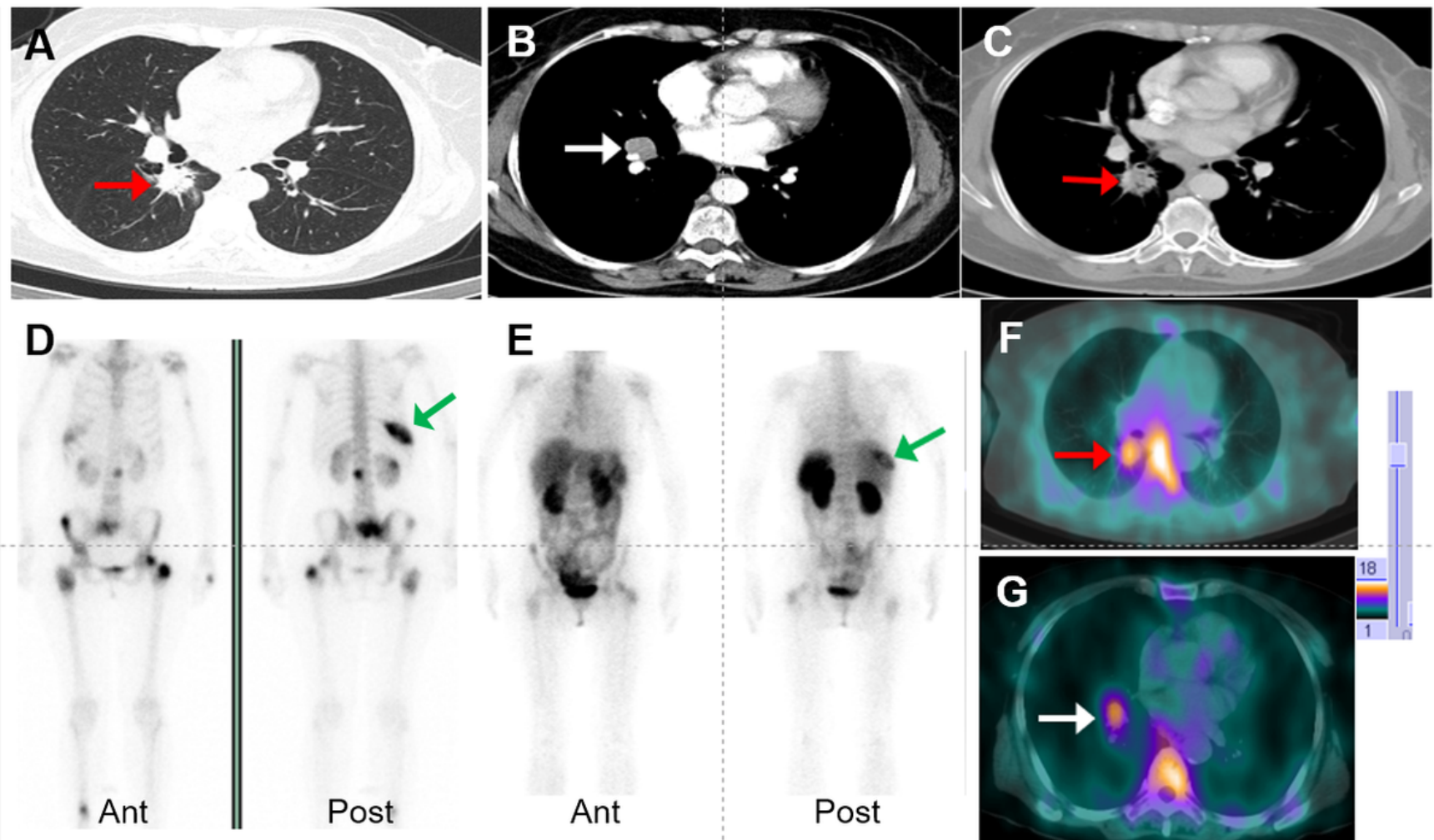

E
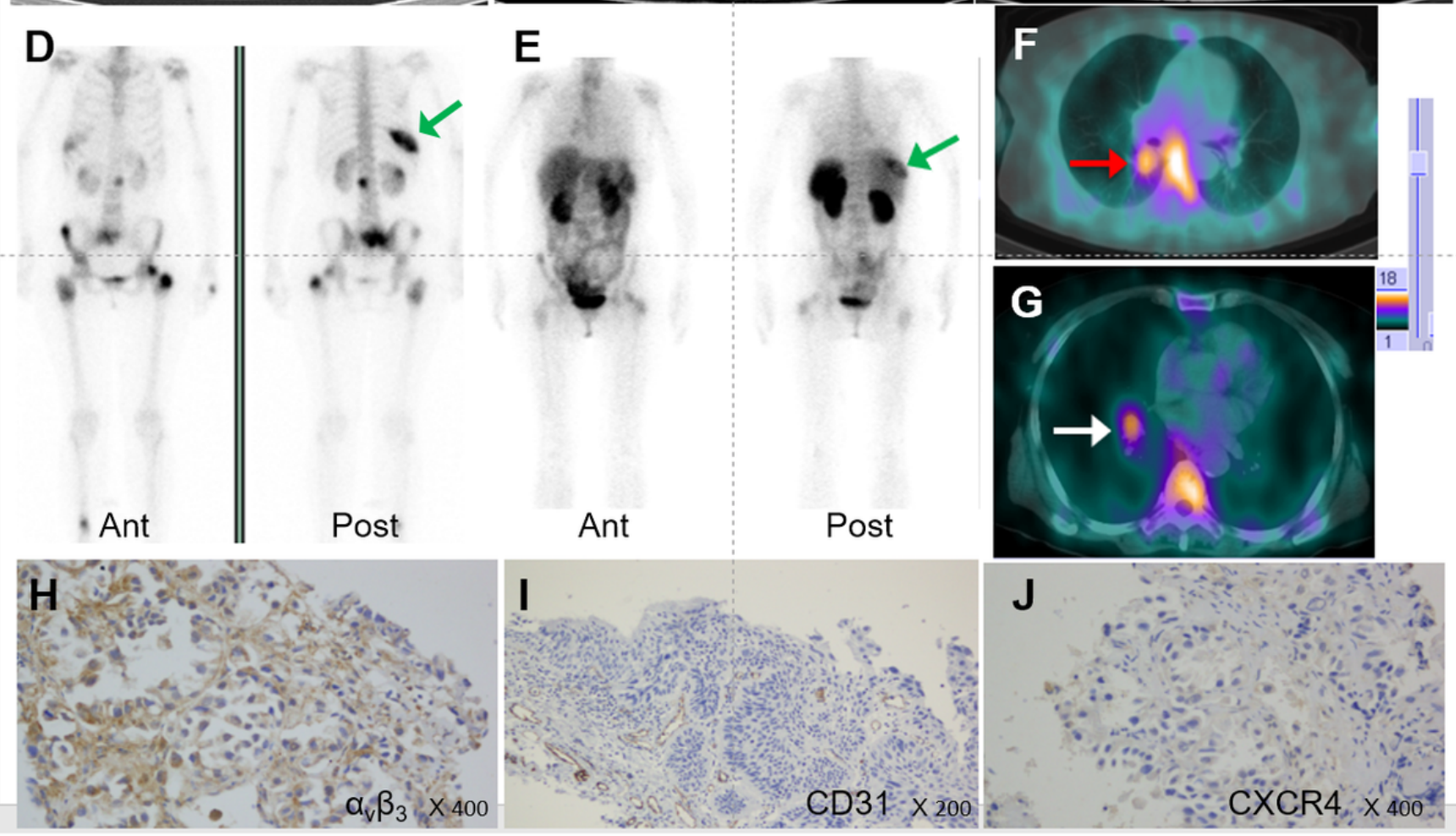
Figure 2

99mTc-Galacto-RGD2 imaging showed RGD-avid uptake in the primary tumour, lymphadenopathy, and remote metastases in a patient with suspected multiple myeloma. The final diagnosis was lung adenocarcinoma confirmed by bronchoscopic biopsy. (A): Primary lesion presented in the lung window. (B, C) Enhanced CT showed primary tumour and lymphadenopathy in the right hilum. (D, E): Bone scan showed lytic lesion in the right rib and sclerotic lesions in the pelvis. $(F, G)$ : 99mTc-Galacto-RGD2 image showed avid lesions in the right lung, hilum, and right rib. (H, I, J): IHC staining. (H) av 33 expression. (I) CD31 expression in neo-vasculature. J: CXCR4 expression in tumour tissue (Zoom:200).



Figure 3

Ratio of primary tumour to normal tissue (T/NT) in different groups. ${ }^{*} p<0.05,{ }^{* \star} p<0.01,{ }^{* \star *} p<0.001$ 

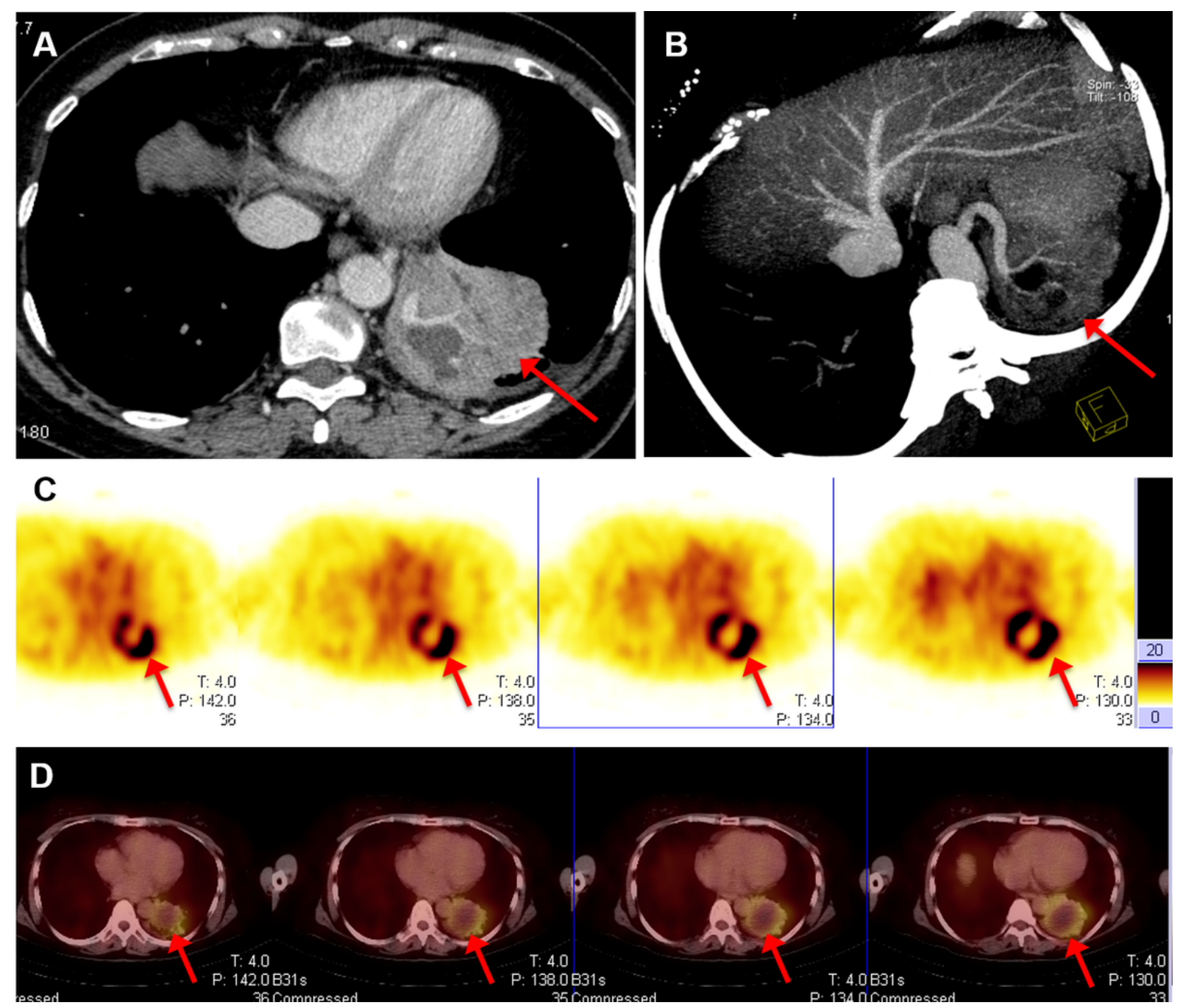

Figure 4

99mTc-Galacto-RGD2 imaging showed focal uptake in a patient with pulmonary sequestration. (A, B) Enhanced CT showed the tumour blood supply originated from the abdominal aorta. (C) RGD imaging showed focal uptake in the tissue, and necrosis in the central zone of the tissue. 

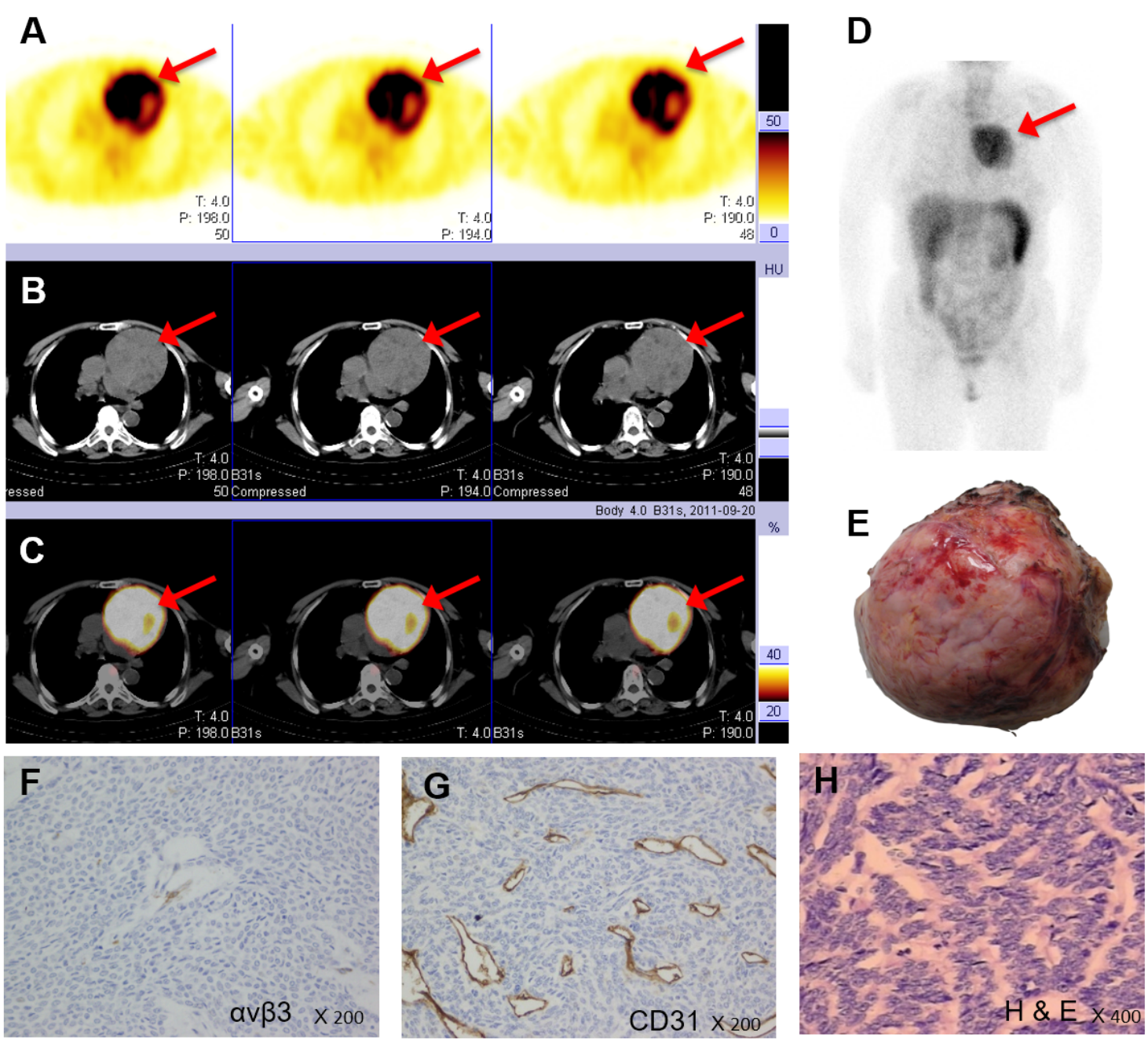

\section{Figure 5}

Female patient with an anterior mediastinum lesion, the final diagnosis was thymus adenoma.『A, B, C, D凶99mTc-Galacto-RGD2 imaging showed high focal

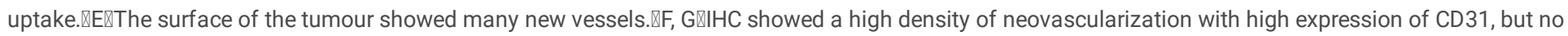
significant av $\beta 3$ expression in the tumour cell membrane.『H囚H\&E staining. 


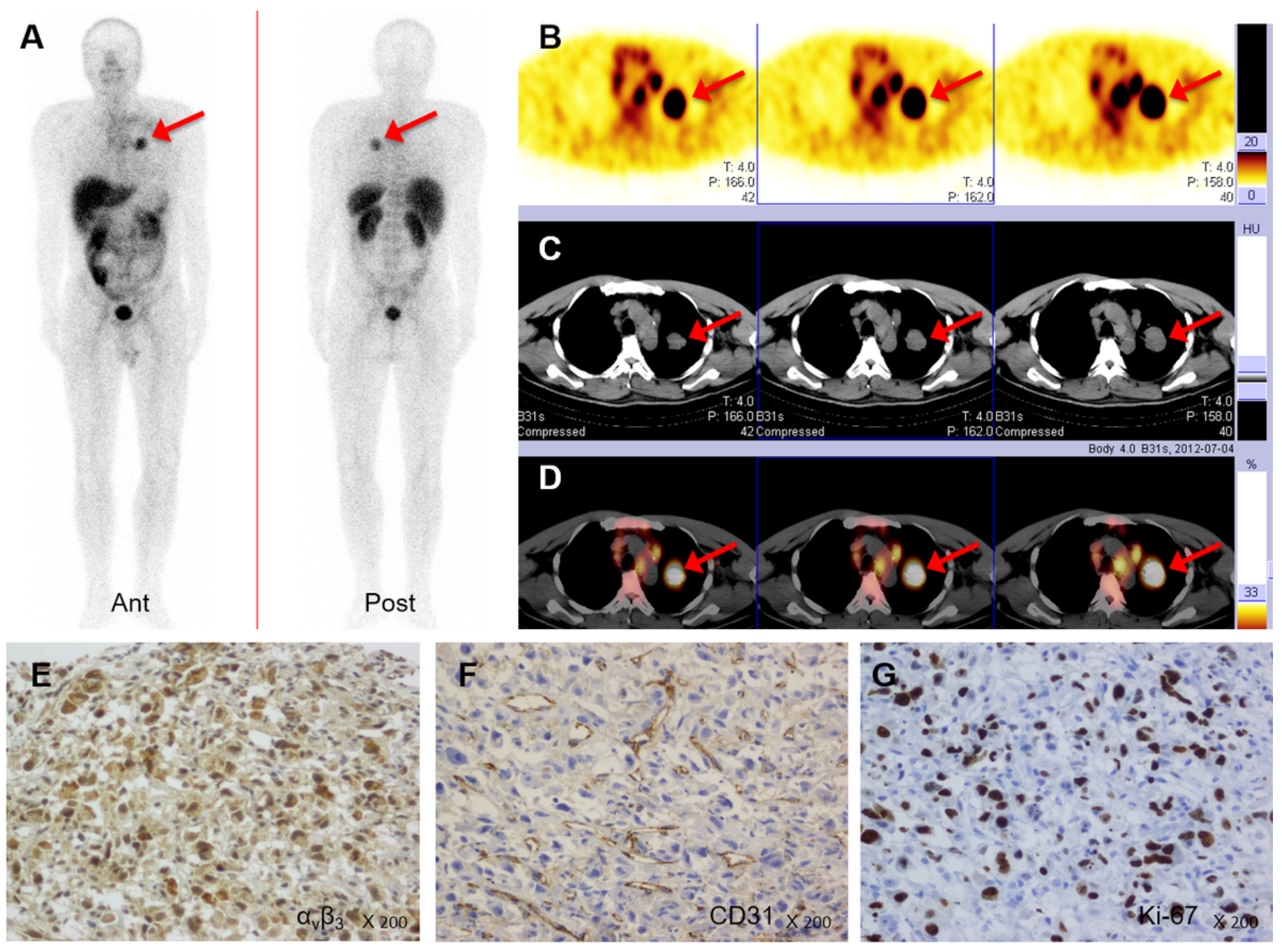

\section{Figure 6}

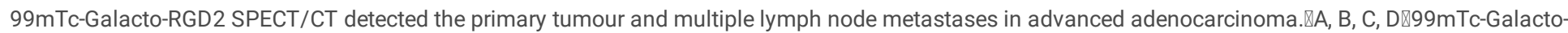
RGD2 image. A: Whole-body image. (B, C, D): SPECT/CT.『E, F, G『: IHC showed higher expression of av $\beta 3$ in the tumour cells and neo-vasculature, higher density of micro-vessel with CD31 expression in the tumour tissue, and a higher Ki-67 index.

\section{Supplementary Files}

This is a list of supplementary files associated with this preprint. Click to download.

- Figuresupplementfinal1.pptx 